\title{
ORIGINAL
}

\section{INCIDENCIA DE TUBERCULOSIS RESPIRATORIA EN LA PROVINCIA DE LEÓN SEGÚN EL SISTEMA DE NOTIFICACIÓN DE ENFERMEDADES DE DECLARACIÓN OBLIGATORIA, 1992-1999}

\author{
Vicente Martín (1), María Aránzazu Alonso (1), Julio Ramos (2), Arancha Otero (3), José \\ Cortizo (3) y Santiago Travieso (2). \\ (1) Área de Medicina Preventiva y Salud Pública. Departamento de Fisiología. Universidad de León. León. \\ (2) Servicio Territorial de Sanidad y Bienestar Social. Junta de Castilla y León. León. \\ (3) Servicio de Cartografía. Universidad de León. León.
}

\section{RESUMEN}

Fundamento: La tuberculosis continúa constituyendo un importante problema de salud pública. Conocer la incidencia, evolución y distribución de la tuberculosis declarada puede servir para valorar la influencia de las medidas de prevención y control puestas en marcha y apuntar nuevas necesidades.

Métodos: Estudio retrospectivo de los casos declarados de TB pulmonar en la provincia de León, recogidos por año, Áreas de Salud (AS) y Zonas Básicas de Salud (ZBS) en el periodo comprendido entre los años 1992 a 1999, ambos inclusive.

Resultados: Las tasas de incidencia media anual fueron de 40,$3 ; 38,6$ y 44,4 por 100.000 habitantes y año $\left(10^{5} \mathrm{~h} / \mathrm{a}\right)$ para la provincia de León (PL), Área de Salud de León (ASL) y Área de Salud del Bierzo (ASB) respectivamente. El 70\% fueron varones y el $30 \%$ mujeres. Las tasas específicas por sexo en la provincia de León fueron de 55,5 en los varones y 22,9 por $10^{5}$ en las mujeres. Las tasas específicas por edad y sexo fueron similares para varones y mujeres hasta los 25 años, partir de esa edad la incidencia en los varones fue mucho mayor. La distribución de la TB pulmonar en la provincia de León fue heterogénea siendo más elevada la incidencia en zonas mineras. En el ASL hubo un predominio de casos en las edades más avanzadas de la vida y en el ASB no se encontraron diferencias entre adultos jóvenes y los mayores de 55 años.

Conclusiones: La provincia de León padece una incidencia de TB superior a la estimada para España. Sería necesario intensificar los sistemas de vigilancia epidemiológica y de medidas de control como tratamientos directamente observados y estudio sistemático de contactos para conseguir una mayor efectividad en el control de esta enfermedad.

Palabras clave: Tuberculosis. Incidencia. Enfermedades infecciosas y parasitarias.

Correspondencia:

Vicente Martín

Área de Medicina Preventiva y Salud Pública.

Departamento de Fisiología.

Campus de Vegazana s/n

24071 León

Correo electrónico: dfivms@unileon.es

\section{ABSTRACT}

Incidence of Respiratory Tuberculosis in the Province of Leon According to

the System for the Compulsory Reporting of Communicable Diseases, 1992-1999

Background: Tuberculosis continues to be a major public health problem. Knowing the incidence, trend and geographical spread of reported tuberculosis may serve to evaluate the prevention and control measures implemented and point to further needs.

Methods: Retrospective study of the reported cases of tuberculosis in the province of Leon, broken down by years, Health Care Areas and Basic Health Care Districts for the 1992-1999 period.

Results: The average annual incidence rates were 40.3, 38.6 and 44.4 per 100,000 inhabitants/year $\left(10^{5}\right.$ inhabitants/year) for the province of Leon, Leon Health Care Area and Bierzo Health Care Area, respectively. Seventy percent $(70 \%)$ were males and thirty percent $(30 \%)$ females. The specific rates by sex in the province of Leon were 55.5 for males and 22.9 per $10^{5}$ for females. The specific rates by age and sex were similar for males and females up to age 25 , the incidence among males being much greater as of this age. The geographical spread of tuberculosis in the province of Leon was heterogeneous, the highest incidence being in mining areas. In the Leon Health Care Area, cases among the oldest adults prevailed, no differences being found among young adults and adults over age 55 in the Basic Health Care District.

Conclusions: The province of Leon has an incidence of tuberculosis greater than that estimated for Spain as a whole. It would be necessary to step up epidemiological surveillance and control measure systems as directly-monitored cases detected and systematic study of contacts in order to achieve a greater efficiency regarding the control of this disease.

Keywords: Communicable diseases. Infections. Tuberculosis. Incidence. 


\section{INTRODUCCIÓN}

La salud pública cuenta con mecanismos muy eficaces para la prevención y el control de la tuberculosis (TB). El diagnóstico precoz de los casos bacilares y su curación bastarían para lograr un efectivo control de la transmisión de la infección. Sin embargo, esta elevada eficacia no se corresponde con una aceptable efectividad, por lo que la TB continúa constituyendo un importante problema de salud pública que ha llevado a la OMS a declarar la situación de emergencia mundial ${ }^{1-3}$. Aunque la mayoría de los casos se producen en los países en vías de desarro1lo, la evolución en los países desarrollados dista de la anunciada erradicación para principios de este siglo ${ }^{1-3}$. La situación de endemia tuberculosa en España no se corresponde con su nivel de desarrollo económico y social ni con sus recursos asistenciales, encontrándose en una situación similar a la que países de su entorno tenían hace 25 años $^{4}$, debido fundamentalmente a problemas de mala adhesión a los tratamientos antituberculosos 5 . La provincia de León, en los años 80, presentaba unas tasas de incidencia de TB declarada cercana a 41 casos por cien mil habitantes, cifras aún superiores a la media nacional ${ }^{6}$.

El objetivo de este trabajo es conocer la incidencia, evolución y distribución de la TB pulmonar declarada en nuestra provincia en los últimos años, con el fin de conocer la influencia de las medidas de prevención y control puestas en marcha y apuntar nuevas necesidades.

\section{MATERIAL Y MÉTODOS}

Se ha llevado a cabo un estudio retrospectivo de los datos aportados por el Sistema de Notificación de Enfermedades de Declaración Obligatoria (SNEDOS) que figuran recogidos en la Sección de Epidemiología del Servicio Territorial de Sanidad y Bienestar Social de León desde el año 1992 a 1999, ambos incluidos. Se recogieron los casos declarados bajo el epígrafe Tuberculosis Pulmonar (Rúbrica 011 CIE-OMS 9. ${ }^{\mathrm{a}}$ Revi- sión), y su distribución por año, Área de Salud, Zona Básica de Salud, edad y sexo. La tuberculosis pulmonar es, en Castilla y León, una enfermedad de declaración obligatoria nominal registrándose únicamente los casos domiciliados en la provincia, no existiendo duplicaciones de casos. La declaración es en grado de sospecha clínica y no se dan de baja los casos no confirmados, si bien, casi el $90 \%$ de los casos son declarados por fuentes Hospitalarias y el Dispensario de Enfermedades del Tórax de León y alrededor del $80 \%$ de los casos son confirmados bacteriológicamente o por anatomía patológica en el momento de la declaración. La Declaración Obligatoria de Enfermedades viene regulada en Castilla y León por lo dispuesto en el Decreto $61 / 1985^{7}$ y por la Orden de 1 de agosto de 1985, ambos de la Consejería de Sanidad y Bienestar Social ${ }^{8}$. Todo médico en ejercicio viene obligado a notificar los casos del listado de enfermedades de declaración obligatoria a las Secciones de Epidemiología — en las modalidades de notificación ugente, nominal y numérica-, elaborando dichas Secciones la información correspondiente al conjunto provincial y notificando la misma, con carácter semanal, al Servicio de Epidemiología de la Consejería de Sanidad y Bienestar Social, desde donde, a su vez, es remitida la información de toda la Comunidad Autónoma al Centro Nacional de Epidemiología.

Las tasas anuales de enfermedad por cien mil habitantes y año se han calculado estableciendo en el numerador los casos declarados y en el denominador el Censo Poblacional de 1991 para los años 1992 y 1993 y del Padrón de 1996 para los años comprendidos entre 1994 y $1999^{9,10}$. Las tasas promedio se han obtenido calculando la media aritmética de las tasas anuales.

El cálculo de las tasas específicas por sexo y edad se han realizado en base al $\mathrm{Pa}$ drón de 1996 y sobre los casos declarados entre los años 1993 y 1999; la tasa promedio se ha obtenido mediante el cálculo de la media aritmética de las tasas anuales. La ten- 
dencia fue calculada mediante el Test de Mantel-Haenszel para tendencias ${ }^{11}$.

Desde el punto de vista sociodemográfico, la provincia de León se caracteriza, de forma sintética, por tres aspectos. En primer lugar, por la alta concentración de la población en las dos áreas urbanas más importantes, León y Ponferrada; sobre un total de 510.610 habitantes en 1998 en la provincia, estas dos áreas concentraban a $143.081 \mathrm{y}$ 61.469 personas, respectivamente, lo que supone que el $40 \%$ de la población provincial reside en estos dos municipios; la densidad resultante es de 3.650 habitantes $/ \mathrm{km}^{2}$ en León y 217 en Ponferrada.

En segundo lugar la población de la provincia está fuertemente envejecida, con un índice de vejez de 153,18 para 1996 (153 mayores de 64 años por cada 100 jóvenes de menos de 15 años), por encima de la media de Castilla y León y muy superior a la media nacional. Los casos concretos de los municipios de León y Ponferrada presentan estructuras menos envejecidas: 128,78 y 99,89, respectivamente.

Finalmente, desde la perspectiva económica, la población leonesa presenta sus mayores tasas de ocupación en el sector servicios; sin embargo, en 1997 más de 8.000 trabajadores estaban englobados en el sector extractivo (minería, canteras), sobre todo en el Área de Salud de Ponferrada y más concretamente en los municipios de Villablino, Fabero, Bembibre y Torre del Bierzo; en el Área de Salud de León la importancia numérica es menor y estos trabajadores están más dispersos en el territorio.

La organización sanitaria de la provincia de León está estructurada en dos Áreas de Salud (León: ASL y El Bierzo: ASB) y Zonas Básicas de Salud (27 en el ASL y 10 en el ASB).

\section{RESULTADOS}

En la provincia de León el número de casos declarados de TB pulmonar entre 1992 y
1999 fueron 1.673 , de los cuales, 1.130 pertenecían al ASL y 543 al ASB. La tasa de incidencia anual promedio de TB pulmonar declarada en la provincia de León fue de 40,3 por cien mil habitantes y año $\left(10^{5} \mathrm{~h} / \mathrm{a}\right)$ oscilando desde una tasa de 47,2 por $10^{5} \mathrm{~h} / \mathrm{a}$ en 1992 a 28,4 por $10^{5} \mathrm{~h} / \mathrm{a}$ en 1999 con una tendencia decreciente estadísticamente significativa $(p=0,028)$ (figura 1$)$.

En el ASL la tasa de incidencia anual promedio fue de 38,6 por $10^{5} \mathrm{~h} / \mathrm{a}$, variando desde una tasa de 48,0 por $10^{5} \mathrm{~h} / \mathrm{a}$ en 1992 a 27,7 por $10^{5} \mathrm{~h} / \mathrm{a}$ en 1999 , con una tendencia descendente y estadísticamente significativa $(p=0,001)$ (figura 1). En el ASB la tasa de incidencia anual promedio fue de 44,4 por $10^{5} \mathrm{~h} / \mathrm{a}$, oscilando desde una tasa de 45,1 por $10^{5} \mathrm{~h} / \mathrm{a}$ en 1992 a 30,2 por $10^{5} \mathrm{~h} / \mathrm{a}$ en 1999, con un pico máximo de 63,0 en 1997, no observándose ninguna tendencia estadísticamente significativa $(\mathrm{p}=0,37)$ (figura 1).

La distribución por Zonas Básicas de Salud (ZBS) mostró que Toreno, Bembibre y Matallana de Torío presentaron las tasas de incidencia anual promedio más elevadas, entre 60 y 86 casos por $10^{5} \mathrm{~h} / \mathrm{a}$. Las ZBS de León, Ribera del Esla, Mansilla de las Mulas, Valencia de Don Juan, Valderas, Babia, Villafranca del Bierzo y Puente de Domingo Flórez, registraron una incidencia promedio entre 45 y 60 casos por $10^{5} \mathrm{~h} / \mathrm{a}$. Las ZBS de Astorga II, Santa María del Páramo y Riaño presentaron las tasas más bajas, inferiores a 15 casos por $10^{5} \mathrm{~h} / \mathrm{a}$. (figura 2 ).

Desde el año 1993 a 1999 se declararon 1.402 casos, de los cuales, 980 (70\%) eran varones y 422 mujeres (30\%). Esta proporción se mantiene para las dos Áreas de Salud. Las tasas de incidencia anual promedio específicas por sexo en la provincia fueron de 55,5 por $10^{5} \mathrm{~h} / \mathrm{a}$ para los varones y de 22,9 por $10^{5} \mathrm{~h} / \mathrm{a}$ para las mujeres. En el ASL fueron de 53,0 por $10^{5} \mathrm{~h} / \mathrm{a}$ y 21,9 por $10^{5} \mathrm{~h} / \mathrm{a}$ respectivamente, y en el ASB de 61,1 por $10^{5} \mathrm{~h} / \mathrm{a}$ y 25,5 por $10^{5} \mathrm{~h} / \mathrm{a}$. 


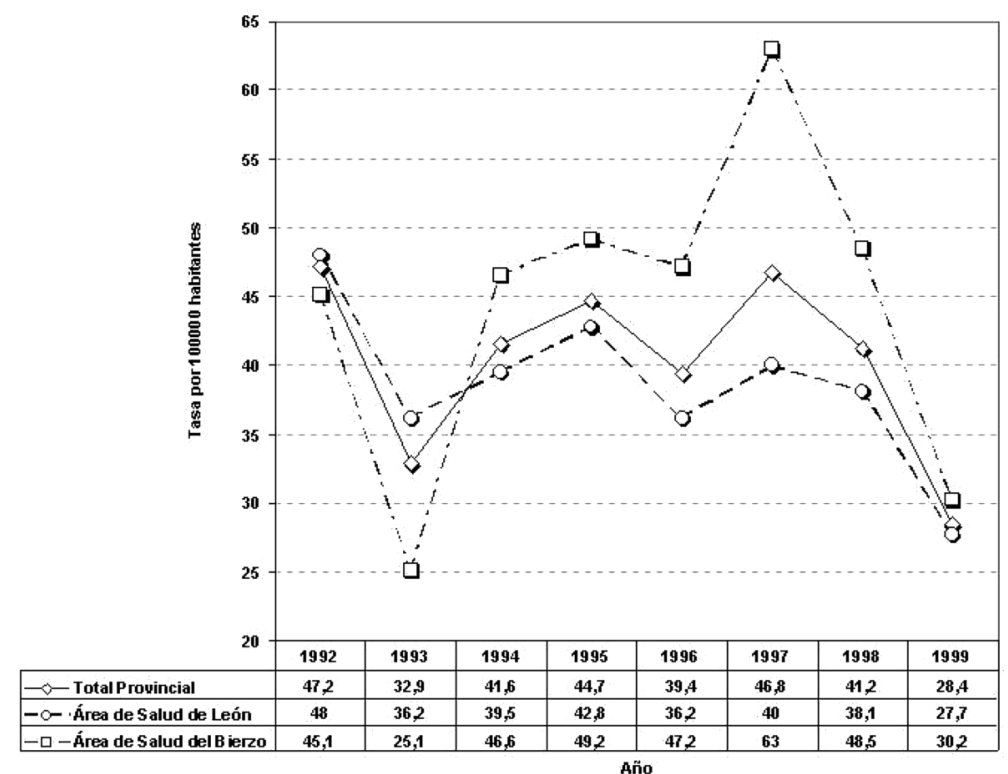

Las tasas de incidencia promedio anual específicas por edad y sexo mostraron tasas similares entre varones y mujeres hasta los 25 años, a partir de esa edad la incidencia en los varones superó ampliamente las tasas de las mujeres (figura 3 ).

En los hombres en el grupo de edad entre 0 y 4 años se observó una tasa promedio de 29,2 por $10^{5} \mathrm{~h} / \mathrm{a}$, un descenso de la misma en el grupo de 5-14 años y posteriormente se incrementó progresivamente con la edad (figura 3). En las mujeres en el grupo de edad entre 0 y 4 años se observó una tasa promedio de 36,3 por $10^{5} \mathrm{~h} / \mathrm{a}$, un descenso en el grupo de 5-14 años y un incremento en los grupos de edad de 15 a 34 años; posteriormente desciende y se mantienen las tasas más bajas entre los 35 y 64 años, a partir de donde se observó un incremento de la tasa (figura 3).

En el ASB las tasas específicas por edad fueron superiores a las del ASL, excepto en los mayores de 64 años (figura 4). Se observó un predominio de casos en las edades más avanzadas de la vida en el ASL y en el ASB no se encontraron diferencias entre las tasas de adultos jóvenes y las de los mayores de 55 años (figura 4).

En la tabla 1 puede observarse como tres cuartas partes de los casos son declarados por fuentes hospitalarias y el resto, casi a partes iguales, por el Dispensario de Enfermedades del Tórax y los Centros de Salud.

\section{Tabla 1}

Distribución de los casos de tuberculosis pulmonar declaradados en la provincia de León entre 1993 y 1999 según categoría de centro declarante

\begin{tabular}{|lrc|}
\hline Centro & Casos & $\%$ \\
\hline Hospitales & 1.073 & 75,3 \\
Dispensario & 168 & 11,8 \\
Centros de salud & 184 & 12,9 \\
\hline Total & 1.425 & \\
\hline
\end{tabular}

Rev Esp Salud Pública 2002, Vol. 76, N. ${ }^{\circ} 3$ 


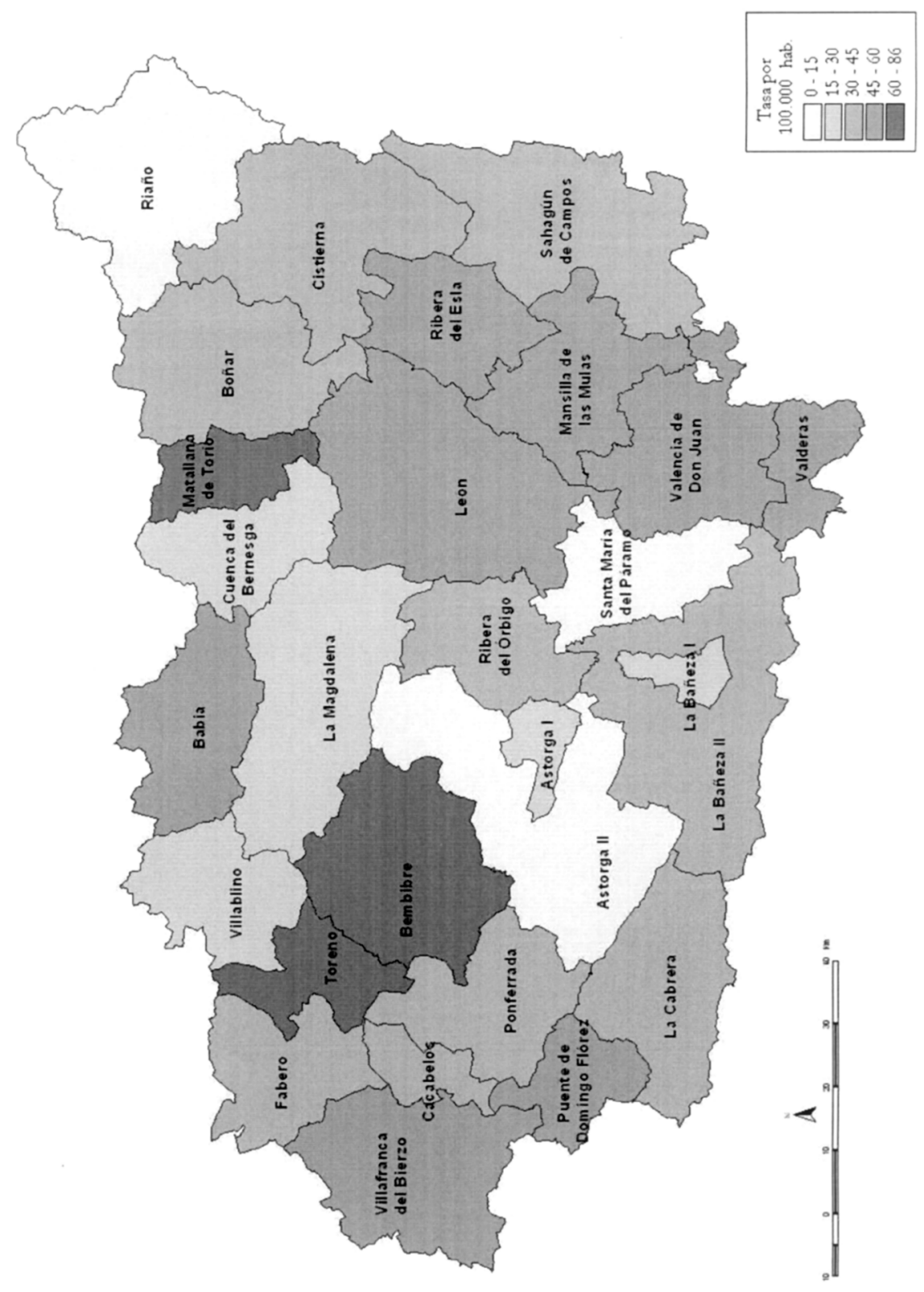


Figura 3

Distribución de la incidencia de tuberculosis pulmonar declarada en la provincia de León según grupos de edad y Área de Salud

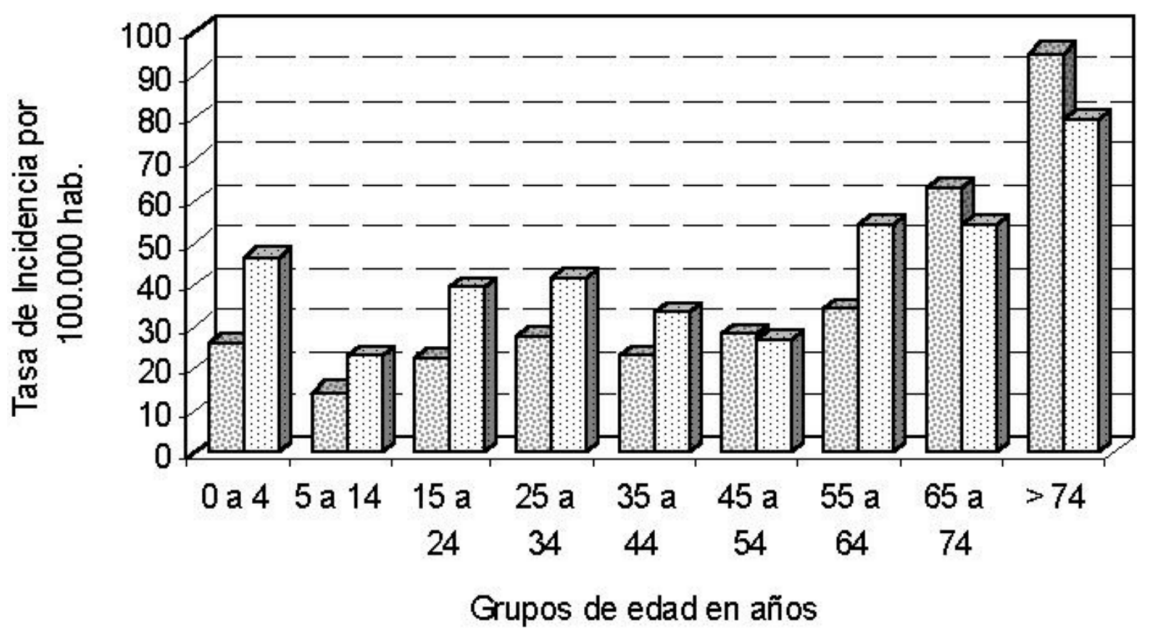

圆Área de León 0 Área del Bierzo

Figura 4

Distribución de la incidencia de tuberculosis pulmonar declarada en la provincia de León según sexo, grupos de edad y Área de Salud

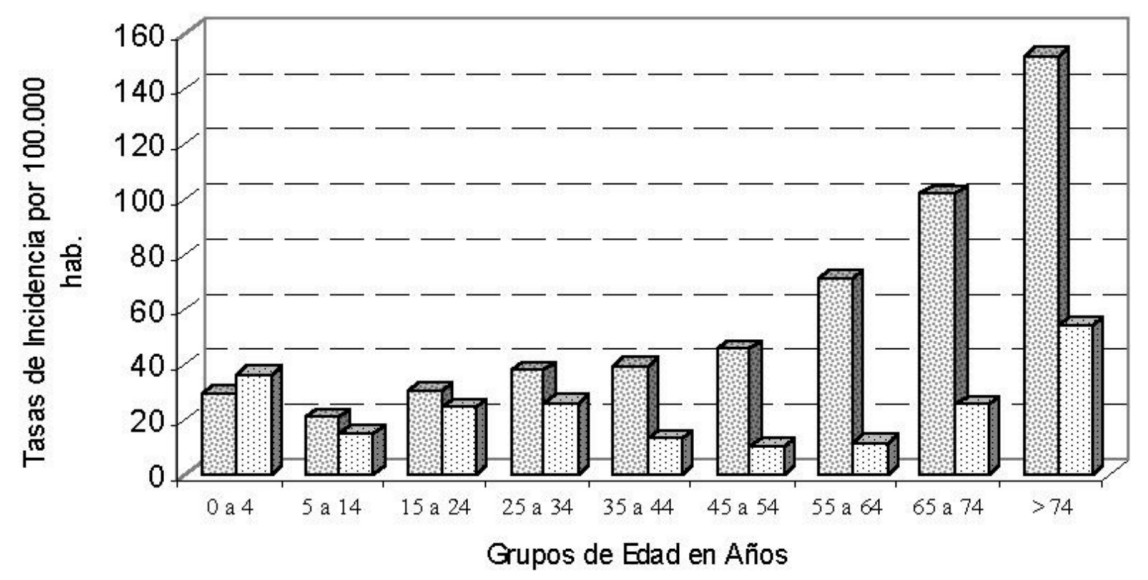

Thombres 0 mujeres 


\section{DISCUSIÓN}

La incidencia promedio de TB declarada en la provincia de León entre 1992 y 1999 $\left(40,3\right.$ casos por $\left.10^{5} \mathrm{~h} / \mathrm{a}\right)$ fue muy similar a la informada para esta provincia en $1988(40,7$ casos por $\left.10^{5} \mathrm{~h} / \mathrm{a}\right)^{6}$ y ligeramente inferior a la encontrada por el Proyecto Multicéntrico de Investigación en Tuberculosis (PMIT) para la provincia $\left(47,54\right.$ casos por $\left.10^{5} \mathrm{~h} / \mathrm{a}\right)$, si bien en este estudio se incluían todas las formas de $\mathrm{TB}^{12}$. Estos hallazgos son sugerentes de un adecuado cumplimiento en la notificación de casos con una cobertura relativamente buena y poca infradeclaración, dado lo exhaustivo de la búsqueda activa del proyecto PMIT.

La tasa promedio observada fue superior a la declarada en la Comunidad Autónoma entre 1992 y 1999, donde osciló entre 34,2 y 21,2 casos por $10^{5} \mathrm{~h} / \mathrm{a}^{13}$ y a la observada por el PMIT en esta Comunidad Autónoma $\left(36,4 \text { casos por } 10^{5} \mathrm{~h} / \mathrm{a}\right)^{14}$.

La incidencia promedio observada fue similar a la estimada por el grupo TIR para nuestro país (40-50 casos por $\left.10^{5} \mathrm{~h} / \mathrm{a}\right)^{15} \mathrm{y}$ similar a la observada por el PMIT $(38,5$ por $10^{5} \mathrm{~h} / \mathrm{a}$ ) para España para todas las TB, si bien en dicho trabajo se encontraron importantes variaciones entre Comunidades Autónomas, desde 14,3 casos por $10^{5} \mathrm{~h} / \mathrm{a}$ en Castilla La Mancha a 70,8 casos por $10^{5} \mathrm{~h} / \mathrm{a}$ en Galicia.

La tendencia decreciente observada es fruto fundamentalmente del importante descenso observado en el año 1999 en los casos declarados y no permite afirmar que efectivamente se haya producido un descenso significativo en las tasas de la enfermedad. Es preciso observar la incidencia en los próximos años para valorar este hallazgo, máxime cuando la tendencia descendente se debe exclusivamente a lo observado en el área de León (La tendencia descendente parece confirmarse en los años 2000 y 2001 con 147 y 144 casos declarados respectivamente $\mathrm{y}$ unas tasas de incidencia de 30,80/100000 y
28,40/100000). Algunos programas han producido un descenso en la incidencia de TB, probablemente asociado a los tratamientos antirretrovirales de alta eficacia y a la mejora de los programas de prevención y control; pero esto sucede en lugares donde la influencia de la infección VIH en la epidemia de TB ha sido importante y/o se han puesto en marcha programas de terapia directamente observada ${ }^{16,17}$. En aquellas zonas donde la influencia de la infección VIH no es tan importante, como Galicia, el descenso observado, como en nuestro caso, es sólo en el último año estudiado, 1998, lo que dificulta la interpretación de los resultados ${ }^{18}$.

Es un hecho conocido cómo la distribución de la TB es heterogénea y cómo depende de condiciones socio-económicas; así se observó que la tasa del ASL (un Área que mezcla zona urbana, la ciudad de León y su alfoz, con zona semiurbana, La Bañeza y Astorga y zonas rurales despobladas y envejecidas) fue de 38,6 casos por $10^{5} \mathrm{~h} / \mathrm{a}$, tasas muy similares a las nacionales; sin embargo, el ASB presentó tasas superiores a las promedio nacionales y regionales, 44,4 casos por $10^{5} \mathrm{~h} / \mathrm{a}$; el hecho de tratarse de una zona donde la minería es una importante actividad económica, puede explicar en alguna medida las diferencias observadas. Abunda en esta idea el que un estudio de casos de TB en el principal Hospital de esta comarca, encontrara que el $23 \%$ de los casos se presentaran en mineros con neumoconiosis $^{19}$.

En el caso de la distribución por Zonas Básicas de Salud también se observó una distribución heterogénea, si bien en este caso, el pequeño tamaño poblacional de muchas de ellas obliga a un prudente análisis; sin embargo se observó que las zonas con tasas de incidencia superiores a 45 casos por $10^{5} \mathrm{~h} / \mathrm{a}$ coincidían con zonas fundamentalmente mineras (Bembibre, Toreno y Matallana de Torio) y las zonas con incidencias inferiores a 15 casos por $10^{5} \mathrm{~h} / \mathrm{a}$, con zonas rurales despobladas (Montaña de Riaño, y la Maragatería). Las zonas de incidencia me- 
dia (entre 45 y 60 casos por 100000 habitantes y año) coincidieron con la ciudad de León y su alfoz que incluye, junto a esta zona eminentemente urbana, núcleos rurales o bien zonas rurales despobladas y con una pirámide de edad envejecida.

En lo que se refiere a la distribución específica por sexos se observó cómo la incidencia de TB es mucho más alta en varones que en mujeres, suponiendo los varones casi las tres cuartas partes de los casos de TB pulmonar declarados. También se observó cómo la incidencia en los varones y las mujeres fue similar hasta la edad adulta, momento a partir del cual fue muy superior en los hombres respecto a las mujeres. Estos datos concuerdan con los obtenidos en el PMIT en nuestro País y en todas las Comunidades Autónomas. Una posible explicación es que los varones a partir de la edad adulta tienen, con mayor frecuencia, factores de riesgo para el desarrollo de la TB una vez infectados (neumoconiosis, tabaquismo, uso de drogas, infección VIH, etc.) que las mujeres.

La distribución de las tasas de incidencia por edades puso de manifiesto cómo el grupo de edad más afectado era el de mayores de 74 años, personas que por mayor edad tienen mayor riesgo de infección por el paso del tiempo y por haber vivido en épocas donde la incidencia de la enfermedad era más elevada y la exposición a fuentes bacilares mayor. También una mayor edad supone una menor competencia inmune y una mayor probabilidad de desarrollar la enfermedad por reactivación endógena ${ }^{20}$. Se observaron también tasas de incidencia muy elevadas en los adultos jóvenes, lo que es concordante con la situación epidemiológica general en nuestro país e indicativo de una importante transmisión reciente y un insuficiente control de la enfermedad ${ }^{4}$. Esta última situación es especialmente notoria en el ASB donde la incidencia en población adulta joven es similar a la de la población de más edad, incluso las elevadas inciden- cias en la población infantil, 0 a 4 años, abunda en esa idea.

La situación epidemiológica de la TB en la provincia de León, al igual que la situación general en nuestro país, está muy alejada de la de los países de nuestro entorno y con recursos sociales y sanitarios similares al nuestro. Esta situación no es compatible con el hecho de que esta enfermedad pueda ser controlada con medidas muy costo-eficientes, como puede ser la estrategia DOTS de la OMS. Esta situación de mal control de la TB es más marcada en las ZBS de zonas mineras; en el Área de Salud del Bierzo y en la capital y su alfoz, lugares en los que pudiera ser de interés establecer la citada estrategia u otros mecanismos de control, recogidos tanto en los Protocolos de la Red Nacional de Vigilancia Epidemiológica ${ }^{21}$ como en el Programa de Prevención y Control de la Tuberculosis de Castilla y León ${ }^{22}$, algunos de los cuales no han sido puestos en marcha o que, habiéndolo sido, posiblemente serían susceptibles de mejora en su cumplimiento. Las principales actividades a llevar a cabo incluirían el perfeccionamiento de los sistemas de vigilancia epidemiológica, de tal modo que permitieran conocer los casos antiguos que son nuevamente declarados, a causa de recaída o de incumplimiento terapéutico, así como el retraso diagnóstico y el seguimiento de los casos diagnosticados para saber cuántos finalizan los tratamientos y se curan. Debería plantearse la necesidad de establecer sistemas de control de los tratamientos a través de tomas supervisadas o directamente observadas, sino en todos los casos, sí al menos en aquellos que se espere una pobre adhesión a los mismos ${ }^{23}$, y la búsqueda de contactos con los casos bacilares ${ }^{24}$.

En conclusión, la provincia de León padece una incidencia de enfermedad tuberculosa superior a la estimada para España, donde la situación ya es considerada no aceptable para nuestro desarrollo social y económico. El Área de Salud de El Bierzo y algunas Zonas Básicas del Área de Salud de León presentan una incidencia aún más elevada. La 
distribución por edades muestra también una transmisión reciente de la infección y un déficit en el control de la enfermedad. Se estima necesario intensificar los sistemas de vigilancia epidemiológica, fundamentalmente conocer el porcentaje de enfermos que finalizan los tratamientos y se curan. Con base a los resultados obtenidos se podrían recomendar estrategias de tratamientos directamente observados o supervisados en los casos de mayor riesgo de incumplimiento.

\section{BIBLIOGRAFÍA}

1. Raviglione MC, Dye C, Schmidt S, Kochi A. Assesment of worldwide tuberculosis control. WHO Global Surveillance and Monitoring Project. Lancet 1997; 350: 624-9.

2. Dye C, Scheele S, Dolin P, Pathania V, Raviglione MC. Global burden of tuberculosis: Estimated Incidence, Prevalence and Mortality by Country. JAMA 1999; 282: 677-686.

3. Lauzardo M, Ashkin D. Phthisiology at the Dawn of the New Century. A review of Tuberculosis and the Prospects of Its Elimination. Chest 2000; 117: $1455-1473$

4. Grupo de Estudio del Taller de 1999 de la Unidad de Investigación en Tuberculosis de Barcelona. Documento de consenso sobre tratamientos directamente observados en Tuberculosis. Madrid: Ministerio de Sanidad y Consumo; 2001.

5. Galdós-Tangüis H, Caylà JA, García de Olalla $P$, Jansà JM, Brugal MT. Factors predicting non-completion of tuberculosis treatment among HIV-infected patients in Barcelona (1987-1996). Int J Tuberc Lung Dis 2000; 4: 55-60.

6. Álvarez Guisasola F, Franch Nadal J, Diego Domínguez F, Alvarez Torices J.C, Álvarez Fernández J.L. La tuberculosis en León. Aproximación epidemiológica. Aten Primaria 1990; 7: 611-4.

7. Decreto $61 / 1985$ de 20 de junio por el que se regula la Declaración Obligatoria de Enfermedades en el territorio de Castilla y León. BOCYL de 2 de julio de 1985 .

8. Orden de la Consejería de Sanidad y Bienestar Social de 1 de agosto de 1985 por la que se aprueba el listado de las enfermedades de Declaración Obligatoria en la CCAA y las modalidades y pro- cedimientos de notificación de las mismas. BOCYL de 22 de agosto de 1985.

9. Nomenclátor del Censo de población de la provincia de León. 1991. Madrid: Instituto Nacional de Estadística; 1993.

10. Nomenclátor del Padrón municipal de habitantes de la provincia de León. 1996. Madrid: Instituto Nacional de Estadística; 1998.

11. Dean AG, Dean JA, Coulombier D, Brendel KA, Smith DC, Burton AH, et al. Epi info Version 6: A Word Processing, Database, and Statistics Program for Epidemiology on Microcomputers. Atlanta: Centers for Disease Control and Prevention; 1994.

12. Documento Interno (no publicado) del Servicio de Epidemiología de la Comunidad Autónoma de Castilla y León. Elaborado en 1999.

13. Archivo histórico de datos EDOS (1979-2000). Sección de Epidemiología de León.

14. Grupo de Trabajo del Proyecto Multicéntrico de Investigación sobre Tuberculosis (PMIT). La tuberculosis en España: resultados del PMIT. Madrid: Instituto de Salud Carlos III; 1999.

15. Rey R, Ausina V, Casal M, Caylà JA, de March $\mathrm{P}$, Moreno S, et al. Situación de la tuberculosis en España. Una perspectiva en precario respecto a los países desarrollados. Med Clin (Barc) 1995; 105: 703-707.

16. Programa de prevención y control de la tuberculosis en Barcelona. Informe 2000. Barcelona: Ayuntamiento de Barcelona; 2001.

17. Comunidad de Madrid. Informe del Registro de Casos de Tuberculosis 1998. Bol Epidemiol Comunidad de Madrid 2000; 6: 3-34.

18. Salgueiro M, Zamarrón C, González J, Vilas A, Suárez J, Duran JL, et al. Estudio Epidemiológico sobre Tuberculosis en el área de Santiago de Compostela en 1995, 1996, 1997 y 1998. An Med Interna 2001; 18: 20-23.

19. Mostaza JL, Fernández S, Bahamonde S, Vélez R, García B, González M, et al. Epidemiología de la tuberculosis en el Bierzo. IX Congreso Nacional de Enfermedades Infecciosas y Microbiología Clínica. Enferm Infecc Microbiol Clin. 2000. 18 (Supl 1): 123.

20. Styblo K. Epidemiology of tuberculosis. The Hague: KNCV, Selected papers, 1991.

21. Protocolos de la Red Nacional de Vigilancia Epidemiológica. Madrid: Centro Nacional de Epide- 
miología; 1996. En http://www.isciii.es/unidad /Sgecnsp/centros/cne/infgralcne.html.

22. Programa de Prevención y Control de la Tuberculosis. Valladolid: Junta de Castilla y León; 1999.

23. Campos F, Muñoz F, Umbría S, Méndez C, Nogales MC. Resultados del tratamiento de la tuberculosis inicial en el área sur de Sevilla en un pe- ríodo de 5 años (1994-1998). Arch Bronconeumol 2001; 37: 177-183.

24. Grupo de Estudio de Contactos de la Unidad de Investigación en Tuberculosis de Barcelona. Documento de consenso sobre el estudio de contac-

tos en los pacientes tuberculosos. Med Clin (Barc) 1999; 112: 151-156. 